\title{
Escala Breve de Ansiedad ante la Evaluación Académica (EBAEA-3)
}

\author{
BRIEF SCALE OF ANXIETY OF THE ACADEMIC EVALUATION (EBAEA-3)
}

\section{Dolores Frías-Navarro' ${ }^{1}$, Marcos Pascual-Soler², Eva Broseta-Aguilar ${ }^{3}$, Héctor Monterde-i-Bort ${ }^{4}$, José Soriano-Pastor ${ }^{5}$, Joana Fornés-Vives ${ }^{6}$, María del Castillo Fuentes-Durán ${ }^{7}$, Fernando García-Pérez ${ }^{8}$, Laura Dolz-Serra ${ }^{9}$, Ana D'Ocón-Gimenez ${ }^{10}$}

\begin{abstract}
${ }^{1}$ Universidad de Valencia, M.Dolores.Frias@uv.es; ${ }^{2}$ ESIC Business \& Marketing School, Valencia, marcos.pascual@esic.edu; 3Universidad de Valencia, broae@alumni.uv.es; ${ }^{4}$ Universidad de Valencia, Hector.Monterde@uv.es; ${ }^{5}$ Universidad de Valencia, jose.f.soriano@uv.es; ${ }^{6}$ Universidad de las Islas Baleares, joana.fornes@uib.es; 7Universidad de Valencia, m.castillo.fuentes@uv.es; ${ }^{8}$ Universidad de Valencia, Fernando.Garcia@uv.es; 9Universidad de Valencia, laura.dolz@uv.es; ${ }^{10}$ Universidad de Valencia, ana.ocon@uv.es
\end{abstract}

Recibido: $27 / 02 / 2020$

Aceptado: 20/07/2020

\section{Resumen:}

Los objetivos: analizar las propiedades psicométricas de una escala breve de ansiedad ante la evaluación académica y comprobar si hay diferencias entre los alumnos que cursan una materia del área de metodología o de otras áreas. La muestra está formada por 404 alumnos de Psicología ( $M=21.03$ años, DT $=5.45$ ). El 56.4\% reciben formación en metodología y el $43.6 \%$ en otras áreas. Los resultados señalan que los estudiantes que reciben formación en materias metodológicas informan de un mayor grado de ansiedad ante la evaluación y perciben que tienen menor autoeficacia. Las implicaciones de los hallazgos están vinculadas con la enseñanza de los contenidos de metodología y alerta a los docentes sobre la necesidad de actuar para controlar esas percepciones.

Palabras clave: ansiedad en educación; ansiedad ante la evaluación; medida, fiabilidad; validez

\section{Abstract:}

The objectives: to analyze the psychometric properties of a brief scale of pre-test anxiety and discover whether there are differences between students taking subjects in the area of methodology or in other areas. The sample is composed of 404 Psychology students $(M=21.03$ years, $S D=5.45)$. Of them, $56.4 \%$ receive training in methodology and $43.6 \%$ in other areas. The results show that the students who receive training in methodological subjects report a greater 
degree of pre-test anxiety and perceive themselves as having less self-efficacy. The implications of the findings are linked to the teaching of the methodology contents, and they warn professors about the need to take steps to control these perceptions.

Keywords: anxiety in education, pre-test anxiety, assessment, reliability, validity

\section{Introducción}

La ansiedad ante la evaluación y el rendimiento del alumnado es un tema que ha suscitado mucho interés en la literatura desde hace décadas (ver Pekrun, 2006 y Zeidner, 2007). En términos generales, la ansiedad es la manifestación de una emoción en la que se anticipa amenaza y el individuo se siente y se describe como inquieto, nervioso, tenso, temeroso o excesivamente preocupado por problemas específicos o tal vez indefinidos $y$, además, suelen haber reacciones fisiológicas negativas (Lobo y Campos, 1997; Zeidner, 2007, 2014). Los estudiantes experimentan cierto grado de ansiedad académica y preocupación (estrés académico), relacionado con la situación de evaluación o examen de los contenidos de las materias que forman su plan de estudios debido a la anticipación de consecuencias negativas como el miedo al fracaso, a la evaluación negativa de los otros o a la misma presión de los padres (Putwain, Connors, y Symes, 2010; Zeidner, 2007). Además, se ha comprobado que hay una relación entre la ansiedad ante la evaluación (ansiedad ante los exámenes) y un mayor riesgo de desarrollar un trastorno de ansiedad y de depresión (Leadbeater, Thompson, y Gruppuso, 2012), conductas de riesgo relacionadas con el abuso de sustancias (tabaco, drogas o alcohol) (Tran, Tran, Geghre, Darmon, Rampal, y cols., 2017), una menor autoestima (von der Embse, Jester, Roy, y Post, 2018) y un bajo rendimiento (Chapell, Blanding, Silverstein, Takahashi, Newman, y cols., 2005). La presencia de problemas de ansiedad y estrés se ha encontrado en todos los niveles académicos y muy especialmente entre los estudiantes universitarios donde el trabajo académico es mucho mayor y se trata de un momento evolutivo que implica enfrentarse a muchos cambios en sus vidas como la posible separación de la familia, el inicio de la independencia, los problemas económicos o la incertidumbre ante su futuro profesional (Eisenberg, Gollust, Golberstein, y Hefner, 2007; Pulido, Serrano, Valdés, Chávez, Hidalgo, y cols, 2011; Stallman, 2010). Por todo ello, es necesario que la universidad ofrezca asesoramiento y tratamiento psicológico a su alumnado, así como fomentar los hábitos saludables para poder mejorar la calidad de la enseñanza y, también, la calidad de la vida cotidiana de sus estudiantes (Fernández-Rodríguez, Soto-López, y Cuesta, 2019; Martínez-Otero, 2014).

Por otra parte, los estudios sobre la ansiedad estadística han comprobado que la ansiedad académica se agrava si las asignaturas están dedicadas al estudio de los diferentes contenidos de la metodología de investigación: estadística, psicometría y métodos de investigación (Murtonen 2005; Murtonen y Lehtinen 2003; Onwuegbuzie, 1997; Onwuegbuzie y Daley 1999; Onwuegbuzie y Wilson 2003; Papanastasiou y Zembylas, 2008). En este sentido, la ansiedad experimentada podría no ser uniforme en cada asignatura, sino que dependería de si se trata de materias del área de la metodología de investigación o de áreas no metodológicas como la psicología básica, vinculada al estudio de los procesos cognitivos y emocionales, o las áreas de educación y personalidad, por ejemplo. La denominada ansiedad estadística describe la aprensión que siente el individuo cuando se expone a contenidos que incluye material estadístico o cuando se enfrenta a situaciones de instrucción o contextos evaluativos donde se incluyen material de estadística (Onwuegbuzie y Wilson, 2003; Macher, Paechter, Papousek, 
Ruggeri, Freudenthaler, y cols., 2013; Macher, Paechter, Papousek, y Ruggeri, 2011; Zeidner, 1990).

Los cursos de metodología de investigación (principalmente métodos de investigación y análisis estadístico) están presentes de forma generalizada en todos los currículos de Ciencias Sociales y de la Salud (Beurze, Donders, Zielhuis, de Vegt, y Verbeek, 2013; González, Rodríguez, Faílde, y Carrera, 2016; Gould, 2010). Concretamente, en Psicología y Educación son una parte esencial de la formación académica, ya sea para que el alumnado diseñe la propia investigación empírica o para que pueda leer de forma crítica o activa los datos de los informes o artículos. Además, esos cursos de metodología son caracterizados por los estudiantes como materias cuyos contenidos son más difíciles de aprender que los de las materias no metodológicas y, por lo tanto, se anticipa un mayor fracaso unido a sus consecuencias negativas (Sandoz, Butcher, y Protti, 2017).

Como ya se ha comentado, la ansiedad estadística representa un constructo que hace referencia a los sentimientos de ansiedad que manifiestan los sujetos cuando reciben un curso de estadística, cuando realizan análisis estadísticos o cuando interpretan los resultados estadísticos en cualquier forma y en cualquier contexto y momento (Cruise, Cash, y Bolton, 1985; Onwuegbuzie, DaRos, y Ryan, 1997). Los autores señalan que la ansiedad es una de las más frecuentes e intensas emociones que experimentan gran parte de los alumnos que reciben clases de estadística en sus grados de Ciencias Sociales y de la Salud (Chew y Dillon, 2014; Faber, Drexler, Stappert, y Eichhorn, 2018). Onwuegbuzie y Wilsom (2003) señalan que aproximadamente el $80 \%$ de los alumnos universitarios informan de ansiedad estadística. Se han desarrollado diversos instrumentos para medir la ansiedad estadística destacando el Statistics Anxiety Rating Scale (STARS, Cruise et al.., 1985) y Statistical Anxiety Scale (SAS, Vigil-Colet, Lorenzo-Seva, y Condon, 2008). Nuestra investigación aporta una escala breve de ansiedad ante la evaluación académica (independientemente de si se trata de ansiedad estadística o no) y puede ser una herramienta útil para el profesorado ya que le podría ayudar en sus tareas diarias en la clase como instrumento para detectar de forma sencilla, ágil y en poco tiempo el estado emocional de su alumnado.

Desde el área de las emociones académicas, es importante considerar el constructo de la ansiedad del alumnado ante la evaluación académica en todo tipo de contexto y no sólo cuando se trata de contenidos de metodología y ansiedad estadística. Los entornos educativos están llenos de experiencias afectivas como ansiedad, diversión, satisfacción, frustración y decepción. $Y$ esas emociones son, en gran medida, resultado de la evaluación de éxito o fracaso académico y de las experiencias personales y sociales agradables o desagradables que el sujeto tiene en sus interacciones educativas (Fiedler y Beier, 2014). Dentro del ámbito de las emociones académicas destaca la denominada ansiedad ante la evaluación académica que representa las emociones y sentimientos que experimenta el alumnado cuando se sienten agobiados al enfrentarse a una situación académica de gran importancia para ellos como puede ser un examen o una prueba de conocimientos (Zeidner, 2014). La ansiedad académica emerge cuando los estudiantes creen que sus habilidades cognitivas y/o motivacionales no podrán superar las demandas que la situación académica requiere para resolver con éxito la evaluación. Evidentemente, el contexto en el que se desarrolla el proceso de enseñanza- aprendizaje es muy relevante cuando se trata de evaluar la ansiedad ante la evaluación académica.

Uno de los entornos que más se ha estudiado es el de la ansiedad experimentada en contextos de aprendizaje de contenidos metodológicos como la estadística o las matemáticas, tal y como ya se ha comentado. Sin embargo, el estudio de la ansiedad ante la evaluación de los contenidos de materias no metodológicas ha sido mucho menor y la investigación se ha orientado más hacia el análisis del estrés percibido por los estudiantes y su calidad de vida (Misra y Castillo, 2004). 
En este sentido, nuestra investigación analiza dos tipos de contexto: aulas donde se enseñan contenidos del área de la metodología de investigación y aulas donde se lleva a cabo la enseñanza de otro tipo de contenidos no específicamente metodológicos.

Muñoz (2004) destaca tres tipos de estresores académicos: 1) estrés relacionado con los procesos de evaluación, 2) estrés relacionado con la sobrecarga de trabajo y 3) estrés relacionado con otras condiciones del proceso de enseñanza-aprendizaje como las relaciones sociales (interacciones entre los alumnos, profesor-alumno), la metodología de enseñanza o diversas cuestiones de organización del plan de estudios y administrativas como horarios, inadecuadas instalaciones o masificación en las aulas. Nuestra investigación se centra en el estresor relacionado con los procesos de evaluación académica y la manifestación de ansiedad directamente relacionada con las actividades de logro o de examen (Spangler, Pekrun, Kramer, y Hofmann, 2002). Y se enmarca en el modelo de la teoría valor-control (control-value theory; Pekrun, 2000, 2006) de las emociones experimentadas en ambientes académicos y de logro. La teoría mantiene que las emociones de logro se inducen cuando el sujeto siente que controla o no controla determinadas actividades y logros que son subjetivamente importantes para él y esas valoraciones de control y valor son los determinantes decisivos de la manifestación de las emociones. El modelo propone que el disfrute de las actividades de logro se fomenta cuando estas actividades se experimentan como controlables y valiosas. Cuando el sujeto percibe que no hay control de las actividades, lo que implica incertidumbre sobre los logros, y concede importancia subjetiva a los resultados, entonces se experimenta ansiedad de logro. Por ejemplo, un estudiante se sentirá ansioso ante un examen si él espera que pueda fallar (hay una certeza subjetiva sobre el fracaso) y percibe el examen como importante.

En nuestro estudio se desarrolla un instrumento breve para medir la ansiedad ante la evaluación académica y se puede aplicar para evaluar el estado emocional del alumnado en todo tipo de materias o asignaturas. Además, el diseño de nuestra investigación analiza un factor potencialmente inductor de estrés académico como es la naturaleza metodológica o no de la materia que se inicia y, por lo tanto, se va a cursar ese año académico (fase 1 del estudio) o de la que se va a examinar en ese mismo momento el alumnado (fase 2 del estudio). Como ya se ha comentado, los resultados de la literatura sobre la ansiedad estadística señalan la importancia de esa emoción cuando el estudiante recibe formación en métodos de investigación y estadística junto a la percepción de alta dificultad de los contenidos y escasa utilidad para su futuro profesional (Gal, Ginsburg, y Schau, 1997; Murtonen, 2005; Murtonen y Lehtinen, 2003; Papanastasiou y Zembylas, 2008).

La Escala Breve de Ansiedad ante la Evaluación Académica (EBAEA-3, Frías-Navarro, BrosetaAguilar, Pascual-Soler, y Monterde-i-Bort, 2019; Frías-Navarro, Monterde-i-Bort, NavarroGonzález, Molina-Palomer, Pascual-Soler, Perezgonzalez, \& Longobardi, 2018) que se presenta en nuestro trabajo consta de 3 ítems y mide de forma breve la intensidad de la ansiedad que percibe el sujeto ante la evaluación académica que debe afrontar. La escala evalúa la ansiedad en diferentes momentos de aproximación al acto de la evaluación (cuando estudia, cuando piensa en el día del examen y un día antes del examen).

\section{Objetivos}

Los objetivos de nuestro estudio son cinco: 1) presentar una escala breve de ansiedad ante la evaluación académica (consta de 3 ítems), aportando pruebas de evidencia de su validez de constructo analizada con un análisis exploratorio de su estructura y con el análisis de la consistencia interna de sus puntuaciones. 2) Presentar pruebas de evidencia de validez de la 
Escala EBAEA-3 analizando su relación con las variables de ansiedad rasgo, ansiedad estado en situación académica, ansiedad generalizada, percepción de autoeficacia académica y síntomas de depresión. 3) Analizar si hay diferencias estadísticamente significativas en la ansiedad ante la evaluación en un contexto de no evaluación inmediata (fase 1 del estudio: la medida se realiza el primer día de clase del curso académico antes de presentar los contenidos y pautas de la guía docente) entre los alumnos que van a recibir docencia en materias de metodología (grupo de materias de metodología) y los que recibirán docencia en materias no metodológicas (grupo de materias no metodológicas). 4) Comprobar la hipótesis de si hay diferencias estadísticamente significativas entre las puntuaciones medias de la escala EBAEA-3 en función de si el alumno debe enfrentarse de forma inmediata a una evaluación/examen de materias del área de metodología (grupo de materias de metodología) o se trata de alumnos que van a realizar un examen de materias de otras áreas no metodológicas (grupo de materias no metodológicas) (fase 2 del estudio: la medida se realiza justo antes de comenzar el examen). 5) Finalmente, también es objetivo de nuestro estudio comprobar si hay diferencias entre los dos grupos (materias de metodología / materias no metodológicas) en la fase 1 (primer día de clase) en las variables de ansiedad rasgo, ansiedad estado, ansiedad generalizada, autoeficacia académica y síntomas de depresión.

\section{Método}

\section{Participantes}

Por medio de un procedimiento de muestreo no probabilístico, y atendiendo a los criterios de accesibilidad, se constituyó una muestra inicial de 404 alumnos de Psicología con una edad media de 21.03 años (DT $=5.45), 329$ son mujeres $(81.6 \%$ ) y 73 son hombres $(18.4 \%)$, controlando que aproximadamente la mitad iban a cursar una materia de metodología y el resto recibían formación en contenidos no metodológicos. En la muestra inicial, el 56.4\% de los estudiantes reciben su formación en materias de metodología y el $43.6 \%$ están cursando asignaturas de otras áreas (personalidad o psicología básica). Esta muestra inicial está formada por los alumnos que asisten el primer día de clase del curso académico a la presentación de los contenidos de la guía docente (fase 1 del estudio). Dicha muestra se reduce a 214 participantes cuando se analizan los datos en el contexto de evaluación académica o examen de la materia, es decir, la medida se realiza justo antes de comenzar el examen final de la materia. En esta segunda fase del estudio la edad media fue 20.58 años (DT = 3.95), 176 son mujeres $(82.2 \%)$ y 38 son hombres (17.8\%). El 50.5\% de los estudiantes reciben su formación en materias de metodología y el $49.5 \%$ están cursando asignaturas no metodológicas. El diseño del estudio es cuasi-experimental ya que incluye una intervención (recibir clases de metodología vs. no recibirlas) pero la asignación de la condición de tratamiento no es aleatoria.

\section{Instrumentos}

Escala Breve de Ansiedad ante la Evaluación Académica (EBAEA-3, Frías-Navarro y cols., 2019). La escala está formada por 3 ítems que miden el nivel de ansiedad que se anticipa ante la evaluación académica o examen de rendimiento académico, registrando la percepción de ansiedad en tres momentos de aproximación a la situación de evaluación académica. Los ítems hacen referencia a la ansiedad que el alumnado percibe "cuando estudia para un examen", "cuando piensa en el día del examen" y "cuando piensa en el examen un día antes de su realización". La escala de respuesta es de tipo Likert con un formato de respuesta que oscila desde 'no ansioso' (1) hasta 'muy ansioso' (10). La amplitud de la respuesta oscila desde 3 hasta 30. El valor de la consistencia interna de los ítems del alfa de Cronbach es excelente tanto 
cuando se aplica el primer día de clase como cuando se aplica en la propia situación de evaluación, es decir, antes del comienzo del examen tal y como se detalla en el apartado de resultados. La medida de la ansiedad ante la evaluación académica se realiza el primer día de clase del curso académico (fase 1 del estudio) y al final del curso justo antes de comenzar el examen final de la materia (fase 2 del estudio).

Índices de Ansiedad Rasgo y Ansiedad Estado en Situación Académica (ARSE y AESE, FríasNavarro, 2017a). La medida de la Ansiedad Rasgo se realiza con un ítem ("en general, mi nivel de ansiedad en mi vida diaria es..."); este ítem se presenta en primer lugar en el cuestionario como ítem 1 seguido de los tres ítems de ansiedad anticipatoria mencionados anteriormente (EBAEA-3) y, finalmente, un ítem sobre la Ansiedad Estado en Situación Académica que el alumnado debe responder justo antes de comenzar el examen de los contenidos que son objeto de evaluación académica ("en estos momentos, mi nivel de ansiedad es..."). Los dos índices se puntúan con una escala de respuesta de tipo Likert con un formato de respuesta que oscila desde 'no ansioso' (1) hasta 'muy ansioso' (10), siendo posible puntuar entre 1 y 10 en cada caso. La medida de los dos índices se realiza el primer día de clase del curso académico (fase 1 del estudio) y al final del curso justo antes de comenzar el examen final de la materia (fase 2 del estudio).

Escala de valoración del trastorno por Ansiedad Generalizada (Generalized Anxiety Disorder, GAD-7; Spitzer, Kroenke, Williams, y Löwe, 2006). La escala GAD-7 consta de 7 ítems que representan siete síntomas de ansiedad. El sujeto tiene que responder con qué frecuencia ha sentido las siguientes sensaciones en los últimos 15 días: nervioso, sin poder controlar la preocupación, muy preocupado por diferentes cosas, con problemas para relajarse, se ha sentido agitado/impaciente, enojado fácilmente y con miedo como si algo terrible fuera a suceder. La escala de respuesta es de tipo Likert con un formato de respuesta con cuatro opciones que oscila desde 'nunca' (0) hasta 'casi todos los días' (3). La amplitud de las respuestas oscila de 0 a 21 y a mayor puntuación más síntomas de ansiedad. La consistencia interna de los ítems de la escala es óptima (alfa de Cronbach $=.88,95 \%$ IC: .87 a .90 ) y la correlación media del ítem con la puntuación total de .52. La medida de la ansiedad generalizada únicamente se realiza el primer día de clase del curso académico.

Autoeficacia académica (Frías-Navarro, 2017b). La autoeficacia académica se ha medido con dos ítems: ¿en qué grado confías en que aprobarás esta materia que ahora recibes? y ¿crees que superarás sin grandes dificultades los contenidos de esta materia que ahora recibes? La escala de respuesta es de tipo Likert con un formato de respuesta que oscila desde 'no ansioso' (1) hasta 'muy ansioso' (10). La amplitud de la respuesta oscila desde 2 hasta 20.Los dos ítems se suman y se utilizan en el estudio como una puntuación total de autoeficacia académica. Cuanto mayor la puntuación mayor es la autoeficacia académica percibida. La correlación entre los dos ítems es alta, $r=.64, p<.001$, y el Intervalo de Confianza del 95\%: .57 a .69. La medida de la autoeficacia académica únicamente se realiza el primer día de clase del curso académico (fase 1 del estudio).

Síntomas de depresión (Patient Health Questionnaire-2, PHQ-2, Kroenke, Spitzer, y Williams, 2003). La sintomatología depresiva se ha medido con dos ítems y el sujeto debe valorar con qué frecuencia ha sentido las siguientes emociones en los últimos quince días: poco interés o placer por hacer las cosas y depresión o desamparo. La escala de respuesta es de tipo Likert con un formato de respuesta con cuatro opciones que oscila desde 'nunca' (0) hasta 'casi todos los días' (3). La amplitud de las respuestas oscila de 0 a 6 y a mayor puntuación mayor es la percepción de sintomatología depresiva. Los dos ítems se suman y se utilizan en el estudio como una puntuación total de sintomatología depresiva. La correlación entre los dos ítems es alta, $r=.72$, 
$\mathrm{p}<.001$, y el Intervalo de Confianza del 95\%: .67 a .76. La medida de la sintomatología depresiva únicamente se realiza el primer día de clase del curso académico (fase 1 del estudio).

\section{Procedimiento}

La administración de los diferentes instrumentos de medida se realizó en horario de clase y se completaron de forma individual en formato auto-administrado en 7 clases diferentes. La participación fue voluntaria tras haber recibido información sobre el propósito y la naturaleza del estudio y se garantizó el anonimato. Se les indicó que el principal objetivo del estudio era conocer cómo se siente y así poder mejorar la docencia teniendo en cuenta dicha información. De la participación en el estudio no se derivaron beneficios académicos para los estudiantes. Se realizó una primera evaluación el primer día del curso académico (principios de septiembre) durante la presentación de la guía docente, tanto en la clase de las materias de metodología como en la clase de las que tenían contenido no metodológico (fase 1 en contexto de no evaluación académica) y una segunda medición justo antes de comenzar un examen o prueba de evaluación (fase 2 en contexto de evaluación académica). La duración de las pruebas fue aproximadamente de 5-10 minutos el primer día de clase con todos los cuestionarios y de 2-4 minutos el día del examen. No se han detectado diferencias estadísticamente significativas entre las puntuaciones medias de las siete clases que participaron en la variable de ansiedad ante la evaluación académica ni en la fase 1 ni en la fase 2 del estudio. El estudio se desarrolló de acuerdo con los principios de la Declaración de Helsinki sobre investigación con sujetos humanos (World Medical Association, 2013).

\section{Resultados}

Objetivo 1. Validez de constructo y consistencia interna de la Escala Breve de Ansiedad ante la Evaluación Académica (EBAEA-3)

Tabla 1. Resultados del análisis de componentes principales de la Escala EBAEA-3 (intervalo de confianza de alfa del 95\%)

\begin{tabular}{|c|c|c|c|c|}
\hline \multirow[b]{2}{*}{ Ítem } & \multicolumn{2}{|c|}{$\begin{array}{c}\text { Fase } 1 . \text { Contexto de no } \\
\text { evaluación inmediata }\end{array}$} & \multicolumn{2}{|c|}{$\begin{array}{l}\text { Fase 2. Contexto de evaluación } \\
\text { inmediata }\end{array}$} \\
\hline & Componente & Comunalidad & Componente & Comunalidad \\
\hline $\begin{array}{l}\text { 1. Cuando } \\
\text { estudio }\end{array}$ & .918 & .771 & .920 & .721 \\
\hline $\begin{array}{l}\text { 2. Cuando } \\
\text { pienso }\end{array}$ & .888 & .843 & .904 & .847 \\
\hline $\begin{array}{l}\text { 3. Un día } \\
\text { antes }\end{array}$ & .878 & .789 & .849 & .817 \\
\hline $\begin{array}{l}\text { Varianza } \\
\text { explicada }\end{array}$ & $80.06 \%$ & & $79.49 \%$ & \\
\hline Alfa de & .88 & & .87 & \\
\hline Cronbach: & (.85 a .90) & & (.84 a .90) & \\
\hline $\begin{array}{l}\text { Prueba de } \\
\text { Barlett: }\end{array}$ & $\begin{aligned} \chi^{2}(3) & =633.08 \\
p & <.001\end{aligned}$ & & $\begin{array}{c}\chi^{2}(3)=335.94 \\
n<001\end{array}$ & \\
\hline KMO: & $\begin{array}{c}.029 \\
\end{array}$ & & .713 & \\
\hline
\end{tabular}

Como ya se ha explicado, el análisis de la validez de constructo del instrumento y su consistencia interna se ha llevado a cabo en dos momentos: en un contexto de no evaluación académica inmediata ya que se realiza la medición el primer día de clase del curso cuando se presentan los contenidos de la guía docente (fase 1) y en un contexto de evaluación académica inmediata justo antes de comenzar un examen o prueba de evaluación (fase 2). Los resultados de los dos análisis 
exploratorios de componentes principales y rotación varimax llevados a cabo con las puntuaciones de la escala EBAEA-3 en las dos fases de nuestro estudio se detallan en la tabla 1. La prueba de esfericidad de Barlett y la de adecuación de la muestra (medida con Kaise-MeyerOlkin, KMO) señalan que es adecuado llevar a cabo el análisis exploratorio (Frías-Navarro y Pascual-Soler, 2012). Cuando se estima la consistencia interna de la escala (un solo factor) mediante el alfa de Cronbach se comprueba que sus valores son óptimos.

Los resultados del análisis de los ítems de la escala EBAEA-3 en las dos fases de medición de nuestro estudio se detallan en la tabla 2. Cuando se realiza el análisis de ítems, también se observa que los valores de consistencia interna eliminando el ítem con la prueba de alfa de Cronbach no cambian de forma sustancial la consistencia de la escala. La correlación media entre el ítem y la puntuación total es de .70 en la fase 1 y .69 en la fase 2.

Tabla 2. Resultados del análisis de ítems de la Escala EBAEA-3

\begin{tabular}{|c|c|c|c|c|c|c|}
\hline \multirow[b]{2}{*}{ Ítem } & \multicolumn{3}{|c|}{$\begin{array}{l}\text { Fase 1. Contexto de no evaluación } \\
\text { inmediata }\end{array}$} & \multicolumn{3}{|c|}{$\begin{array}{l}\text { Fase 2. Contexto de evaluación } \\
\text { inmediata }\end{array}$} \\
\hline & $\begin{array}{c}\mathrm{M} \\
(D T)\end{array}$ & $\begin{array}{l}\text { Correlación } \\
\text { corregida }\end{array}$ & $\begin{array}{c}\text { Alfa } \\
\text { ítem } \\
\text { eliminado }\end{array}$ & $\begin{array}{c}\mathrm{M} \\
(D T)\end{array}$ & $\begin{array}{c}\text { Correlación } \\
\text { corregida }\end{array}$ & $\begin{array}{c}\text { Alfa } \\
\text { ítem } \\
\text { eliminado }\end{array}$ \\
\hline $\begin{array}{l}1 . \\
\text { Cuando } \\
\text { estudio }\end{array}$ & $\begin{array}{c}5.54 \\
(2.29)\end{array}$ & .73 & .85 & $\begin{array}{c}6.59 \\
(1.88)\end{array}$ & .68 & .88 \\
\hline $\begin{array}{l}2 . \\
\text { Cuando } \\
\text { pienso }\end{array}$ & $\begin{array}{c}5.77 \\
(2.31)\end{array}$ & .80 & .78 & $\begin{array}{c}6.99 \\
(1.94)\end{array}$ & .81 & .77 \\
\hline $\begin{array}{l}\text { 3. Un } \\
\text { día } \\
\text { antes }\end{array}$ & $\begin{array}{c}6.75 \\
(2.23)\end{array}$ & .75 & .84 & $\begin{array}{l}7.37 \\
(1.92)\end{array}$ & .77 & .80 \\
\hline
\end{tabular}

Objetivo 2. Pruebas de evidencia: correlación con ansiedad rasgo, ansiedad estado, ansiedad generalizada, autoeficacia académica, y síntomas de depresión (fase 1 del estudio)

Los resultados de los análisis de correlación entre las puntuaciones de la Escala Breve de Ansiedad ante la Evaluación Académica (ABAEA-3) y las de las variables de ansiedad rasgo, ansiedad estado, ansiedad generalizada, autoeficacia percibida y síntomas de depresión se detallan en la tabla 3.

Tabla 3. Correlaciones e Intervalo de Confianza (95\%) entre las puntuaciones de la Escala EBAEA-3 y el resto de variables psicológicas

\begin{tabular}{lcc}
\hline Variables & $\begin{array}{c}\text { Fase } 1 . \text { EBAEA. Contexto } \\
\text { de no evaluación } \\
\text { inmediata }\end{array}$ & $\begin{array}{c}\text { Fase 2. EBAEA. Contexto } \\
\text { de evaluación inmediata }\end{array}$ \\
\hline EBAEA. Evaluación & $\begin{array}{c}.44 . p<.001 \\
(.33 \mathrm{a} .54)\end{array}$ & - \\
Ansiedad Rasgo & $.42 . p<.001$ & $.50 . p<.001$ \\
Ansiedad Estado & $(.33 \mathrm{a} .49)$ & $. .39 \mathrm{a} .60)$ \\
Ansiedad generalizada & $.31 . p<.001$ & $(.56 \mathrm{a} .73)$ \\
Autoeficacia académica & $(.22 \mathrm{a} .40)$ & $.2001, p=.004$ \\
& $.28 . p<.001$ & $(.07 \mathrm{a} .33)$ \\
Síntomas depresión & $(.19 \mathrm{a} .37)$ & $-.22 . p=.001$ \\
& $-.23 . p<.001$ & $(-.34 \mathrm{a}-.09)$ \\
& $(-.32 \mathrm{a} .-13)$ & $.06 . p=.378$ \\
& $.09 . p=.073$ & $(-.07 \mathrm{a} .19)$ \\
\hline
\end{tabular}


Los valores de los coeficientes de correlación son muy similares en los dos contextos de evaluación académica (fase 1 y fase 2 del estudio) excepto cuando se mide la ansiedad estado en el contexto de justo antes de comenzar a resolver el examen de la materia $(r=.66)$ ya que dobla al valor de la correlación cuando se trata del primer día de clase $(r=.31)$. Es decir, la ansiedad estado de los alumnos en el momento de enfrentarse al examen mantiene una relación directa y muy alta con las puntuaciones de la escala EBAEA-3 de ansiedad ante la evaluación académica. Por lo tanto, el instrumento EBAEA-3 es sensible al estado emocional del alumnado, manifestando así una prueba de su validez de constructo. Además, las puntuaciones de ansiedad ante la evaluación académica en las dos fases correlacionan de forma estadísticamente con la ansiedad generalizada, pero no con los síntomas de depresión. La relación entre ansiedad ante la evaluación y la autoeficacia percibida es inversa y estadísticamente significativa en las dos fases del estudio, es decir, cuando los estudiantes tienen confianza en aprobar y superar la materia experimentan menos niveles de ansiedad ante la evaluación académica.

Objetivo 3. Análisis en una situación de evaluación no académica: materias de metodología vs. materias no metodológicas (fase 1)

Para comprobar si hay diferencias estadísticamente significativas en la ansiedad ante la evaluación en un contexto de no evaluación inmediata (fase 1 ) entre los alumnos que van a recibir docencia en materias de metodología (grupo de materias de metodología) y los que recibirán docencia en materias no metodológicas (grupo de materias no metodológicas) se ha realizado un diseño entre grupos unifactorial univariado. Los resultados señalan que los alumnos que asisten el primer día del curso a una clase donde se van a presentar los contenidos de una materia de metodología (estadística, métodos de investigación, psicometría) obtienen una puntuación media más alta en la escala de ansiedad ante la evaluación académica (EBAEA-3) (M $=18.79, \mathrm{DT}=5.79, \mathrm{n}=228$ ) que los alumnos que se encuentran en una clase donde se expone la guía docente de materias no metodológicas $(M=17.10, D T=6.40, n=176)$, siendo la diferencia estadísticamente significativa y el tamaño del efecto pequeño, $F(1,402)=7.69, p=$ $.006, d=0.28,95 \%$ IC ( 0.08 a 0.48 ). Se comprobó la igualdad de las varianzas de los dos grupos, F-Levene $(1,402)=1.59, p=.208$. Por lo tanto, se comprueba que la ansiedad ante la evaluación académica es más alta en el alumnado que asiste el primer día del curso a una clase donde se van a enseñar contenidos del área de la metodología de investigación, comprobando el efecto del contexto o contenido de las materias. Así, el alumnado anticipa la situación de evaluación de una materia de metodología y expresa un mayor grado de ansiedad ante la evaluación académica.

Objetivo 4. Análisis en una situación de evaluación académica: materias de metodología vs. materias no metodológicas (fase 2)

Cuando la medida se realiza en un contexto de evaluación académica justo antes de comenzar el examen o prueba de evaluación (fase 2 ) se comprueba que los alumnos que se van a examinar de una materia con contenido metodológico también obtienen una puntuación media más alta en la escala de ansiedad ante la evaluación académica (EBAEA-3) $(M=21.72, D T=5.20, n=108)$ que los alumnos que van a realizar un examen de una materia no metodológica ( $M=20.22$, DT $=4.88, \mathrm{n}=106)$, siendo la diferencia estadísticamente significativa y el tamaño del efecto pequeño, $F(1,212)=4,77, p=.03, d=0.30,95 \%$ IC (0.03 a -0.57). Se comprobó la igualdad de las varianzas de los dos grupos, F-Levene $(1,212)=1.07, p=.744$. Por lo tanto, tal y como era de esperar, en una situación de evaluación académica (justo antes de comenzar el examen final de la materia), el alumnado que se enfrenta a un examen de una materia de metodología tiene un nivel más alto de ansiedad en la escala EBAEA-3. 
Objetivo 5. Diferencias el primer día de clase en ansiedad rasgo, ansiedad estado, ansiedad generalizada, autoeficacia académica y síntomas de depresión: materias de metodología vs. materias no metodológicas (fase 1)

Por último, se ha analizado si los dos grupos de alumnos difieren el primer de clase cuando comienza el curso académico (fase 1 ) en las variables de ansiedad rasgo, ansiedad estado, ansiedad generalizada, autoeficacia académica y síntomas de depresión (ver las puntuaciones medias en la tabla 4). En estos análisis se produce la pérdida de las puntuaciones de algunos sujetos porque dejaron algún ítem en blanco. Los resultados señalan que las puntuaciones medias de los dos grupos en la fase 1 no difieren de forma estadísticamente en la variable de ansiedad rasgo, $F(1,402)=1.53, p=.217, d=0.12$, 95\% IC (-0.07 a 0.32$)$; se comprueba que no hay diferencias estadísticamente significativas entre las varianzas de los grupos, F-Levene(1, $402)=1.402, p=.132$; tampoco en la variable de ansiedad generalizada, $F(1,393)=.02, p=.9$, $d=0.01,95 \%$ IC $(-0.19$ a 0.21$)$; se comprueba que no hay diferencias estadísticamente significativas entre las varianzas de los grupos, FLevene $(1,393)=.60, p=.441$, y tampoco en la variable de síntomas de depresión, $\mathrm{F}(1,400)=.1, \mathrm{p}=.752, \mathrm{~d}=0.03,95 \% \mathrm{IC}(-0.17$ a 0.23$)$; se comprueba que no hay diferencias estadísticamente significativas entre las varianzas de los grupos, F-Levene $(1,400)=.84, p=.36$.

Tabla 4. Análisis descriptivo de las variables psicológicas medidas en la fase 1

\begin{tabular}{|c|c|c|c|c|c|c|}
\hline \multirow[b]{2}{*}{ Variable } & \multicolumn{3}{|c|}{$\begin{array}{l}\text { Materias de } \\
\text { metodología }\end{array}$} & \multicolumn{3}{|c|}{$\begin{array}{c}\text { Materias no } \\
\text { metodológicas }\end{array}$} \\
\hline & Media & DT & $n$ & Media & DT & $n$ \\
\hline Ansiedad rasgo & 4.92 & 2.05 & 228 & 4.65 & 2.21 & 176 \\
\hline Ansiedad generalizada & 8.02 & 5.06 & 223 & 7.96 & 4.84 & 172 \\
\hline Síntomas de depresión & 1.49 & 1.56 & 228 & 1.44 & 1.49 & 174 \\
\hline Ansiedad estado & 3.71 & 2.24 & 228 & 3.09 & 2.08 & 176 \\
\hline Autoeficacia académica & 13.65 & 2.9 & 228 & 14.61 & 2.73 & 176 \\
\hline
\end{tabular}

Sin embargo, se comprueba que los dos grupos de estudiantes sí difieren en las variables de ansiedad estado y autoeficacia académica percibida cuando se encuentran en un contexto donde van a recibir la enseñanza de contenidos de una materia de metodología respecto a un contexto de enseñanza de materias de otras áreas no vinculadas con la metodología de investigación (ver tabla 4). En primer lugar, la ansiedad que los alumnos perciben el primer día de clase cuando se presentan los contenidos de la guía docente es mayor en el grupo que va a cursar una materia de metodología que los que se encuentran en un grupo que va a recibir formación en una materia no metodológica, $F(1,402)=7.98, p=.005, d=0.28,95 \%$ IC 0.09 a 0.48 , siendo el tamaño del efecto pequeño; se comprueba que no hay diferencias estadísticamente significativas entre las varianzas de los grupos, F-Levene $(1,402)=1.76, p=$ .185. En segundo lugar, el alumnado que se encuentra en una clase donde el contenido de la materia a cursar es metodológico presentan una puntuación menor en autoeficacia académica que los estudiantes del grupo que van a cursar una materia no metodológica, siendo el tamaño del efecto pequeño, pero ha aumentando, $F(1,402)=11.66, p<.001, d=-0.34,95 \%$ IC $(-0.54$ a -0.14); se comprueba que no hay diferencias estadísticamente significativas entre las varianzas de los grupos, F-Levene $(1,402)<.01, p=.982$. Conviene recordar que la medida de la 
autoeficacia se interpreta como a mayor puntuación mayor autoeficacia y de ahí que el valor del tamaño del efecto sea negativo. Estos dos resultados apoyan el modelo teórico que mantiene mayor nivel de ansiedad ante la evaluación entre los alumnos que reciben contenidos de materias metodológicas en comparación con alumnos que cursan otro tipo de materias cuyo contenido no es metodológico.

\section{Discusión}

Los resultados de nuestra investigación señalan que los estudiantes de Ciencias Sociales y de la Salud que cursan materias de naturaleza metodológica de su plan de estudios tienen un mayor nivel de ansiedad ante la evaluación académica y una menor autoeficacia percibida que los estudiantes que reciben formación en materias no metodológicas. Las implicaciones educativas de estos hallazgos están vinculadas directamente con la enseñanza de los contenidos de metodología de investigación en el ámbito universitario dado el mayor nivel de ansiedad de los alumnos y su menor percepción de eficacia desde el primer día del curso. Además, alerta a los docentes sobre la necesidad de desarrollar instrucciones o planes de actuación que controlen esas percepciones que probablemente interferirán en el clima de la clase y en el rendimiento de los alumnos. En definitiva, el contexto o contenido de la materia que se cursa es relevante cuando se trata de evaluar el nivel de ansiedad académica del alumnado.

Disponer de instrumentos breves es importante para que el profesor pueda llevar a cabo evaluaciones válidas del estado emocional de sus alumnos sin interferir en exceso en las actividades académicas que se desarrollan en las clases. Nuestro estudio aporta un instrumento breve de tres ítems cuyas propiedades psicométricas son adecuadas respecto a su estructura unidimensional y consistencia interna de los ítems y respecto a las pruebas de validez que se aportan en el trabajo.

Los estudiantes universitarios experimentan estrés y ansiedad durante su formación académica ya que tienen que dar respuesta a las demandas de una formación con un alto nivel de exigencia que requiere un grado alto de compromiso personal del alumno para lograr el éxito en las materias que forman su grado. A ello se unen otros estresores como la presión familiar, la falta de apoyo social, los problemas financieros, la competición en las aulas, las relaciones sociales y la inseguridad sobre el futuro (Stallamn, 2010, Tosevski, Milovancevic, y Gajic, 2010). Stallman, Ohan y Chiera (2019) estiman que aproximadamente el $83 \%$ de los estudiantes universitarios tienen trastornos psicológicos. Problemas de ansiedad, depresión e intentos de suicidio han sido estudiados en la literatura dentro del ámbito de los estudios universitarios (Eisenberg y cols., 2007). Poder detectar síntomas de problemas emocionales en el alumnado ayudará a prevenir desajustes más graves o indagar en un posible deterioro en su bienestar cotidiano y tomar decisiones sobre aconsejar algún tipo de intervención. Nuestro trabajo incorpora el estudio de las emociones del alumnado y se enmarca en una línea de investigación que tenemos sobre la innovación educativa en las aulas de la universidad.

Las variables de ansiedad ante la estadística y las actitudes hacia la estadística han sido estudiadas en la literatura desde hace décadas como un foco de estrés percibido por los alumnos que cursan estudios en disciplinas no vinculadas con las matemáticas y que tienen en su plan de estudios materias de metodología de investigación como la estadística o los métodos de investigación donde se aplican las técnicas de inferencia estadística (Bending y Hughes, 1954; Chew y Dillon, 2014; Kesici, Baloğlu, y Deniz, 2011; Nielsen y Kreiner, 2018; Onwuegbuzie y Seaman, 1995). Los resultados de nuestro estudio son una prueba más de los hallazgos detectados en dicha línea de investigación. 
En definitiva, nuestro estudio aporta un instrumento breve de ansiedad cuyo objetivo es medir la ansiedad ante la evaluación académica, independientemente de si el contexto es de formación en materias de metodología o no. La brevedad de la escala y la medida de un constructo más amplio que el de la ansiedad estadística son las dos principales ventajas del instrumento.

La escala EBAEA-3 permite que el profesorado pueda evaluar de forma rápida la situación emocional de sus alumnos, tanto en contextos de evaluación inmediata como en entornos sin evaluación y, así, poder prevenir y planificar actuaciones dirigidas a disminuir y controlar estados emocionales que podrían interferir en el rendimiento y bienestar de los alumnos y contaminar el clima de la clase. La calidad de los resultados de sus propiedades psicométricas y el diseño de nuestro estudio con grupos de metodología y no metodología otorgan calidad al instrumento que discrimina entre los grupos (tanto en un contexto de evaluación inmediata como en contextos sin evaluación) y detecta que los estudiantes que se encuentran realizando un curso con contenidos estadísticos tienen un mayor nivel de ansiedad cuando piensan en la evaluación o examen y, también, perciben un menor nivel de autoeficacia en esos contenidos.

Las principales limitaciones de nuestra investigación son inherentes a la propia naturaleza del diseño de investigación cuasi-experimental aplicado ya que al no existir asignación aleatoria del tipo de contenidos que recibe el alumno hay que tener precaución con las interpretaciones de relaciones causa-efecto entre las variables. La selección de la muestra se realizó por un criterio de accesibilidad al profesorado y su alumnado, pero fue totalmente aleatorio sin ningún tipo de criterio de preferencia externo. Solamente se controló que hubiera participación de clases donde se iba a impartir metodología y clases donde no se iba a impartir metodología. Por lo tanto, consideramos que las relaciones entre la variable de contenido de la materia (metodológico/no metodológico) y la ansiedad ante la evaluación académica pueden interpretarse como causal en los términos de un diseño cuasi-experimental. Sería muy idóneo llevar a cabo este trabajo con un diseño aleatorio donde se pudieran seleccionar las clases de los dos grupos de forma aleatoria a partir de todo el conjunto de clases que forman el plan de estudios.

\section{Agradecimientos}

Al proyecto UV-INV-AE17-698616 financiado por la Universidad de Valencia (España). Y a los alumnos y alumnas que participaron en el estudio y sus profesores y profesoras.

\section{Referencias bibliográficas}

Bendig, A. W. y Hughes, J. B. (1954). Student attitude and achievement in a course in introductory statistics. Journal of Educational Psychology, 45, 268-276.

Beurze, S. M., Donders, A. R. T., Zielhuis, G. A., Zielhuis, G. A., de Vegt, y Verbeek, A. L. M. (2013). Statistics anxiety: A barrier for education in research methodology for medical students? Journal of the International Association of Medical Science Educators, 23, 377-384.

Chapell, M. S., Blanding, Z. B., Silverstein, M. E., Takahashi, M., Newman, B., Gubi, A., y McCann, N. (2005). Test anxiety and academic performance in undergraduate and graduate students. Journal of Educational Psychology, 97, 268-274. 
Chew, P. K. H. y Dillon, D. B. (2014). Statistics anxiety update: Refining the construct and recommendations for a new research agenda. Perspectives on Psychological Science, 9, 196-208.

Cruise, J. R., Cash, R. W., y Bolton, L. D. (1985). Development and validation of an instrument to measure statistical anxiety. En ASA Proceedings (Ed.), Statistical education section (pp. 9298). Washington, DC: American Statistical Association.

Eisenberg, D., Gollust, S. E., Golberstein, E., y Hefner, J. L. (2007). Prevalence and correlates of depression, anxiety, and suicidality among university students. American Journal of Orthopsychiatry, 77, 534-542.

Faber, G., Drexler, H., Stappert, A., y Eichhorn, J. (2018). Measuring education science students' statistics anxiety conceptual framework, methodological considerations, and empirical analyses. Research report. Leibniz University Hannover, Faculty of Humanities, Institute of Psychology.

Fernández-Rodríguez, C., Soto-López, T., y Cuesta, M. (2019). Needs and demands for psychological care in university students. Psicothema, 31, 414-421.

Fiedler, K. y Beier, S. (2014). Affect and cognitive processes in educational contexts. En P. A. Alexander, R. Pekrun, \& L. Linnembrik-García (Eds.), International handbook of emotions in education (pp. 36-55). New York, NY: Routledge.

Frías-Navarro, D. (2017a). Índices de Ansiedad Rasgo en Situación de Examen (ARSE) y Ansiedad Estado en Situación de Examen (AESE). Universidad de Valencia. España.

Frías-Navarro, D. (2017b). Medida de la autoeficacia académica. Universidad de Valencia. España.

Frías-Navarro, D., Broseta-Aguilar, E., Pascual-Soler, M., y Monterde-i-Bort, H. (2019). Escala Breve de Ansiedad ante la Evaluación Académica (EBAEA-3). Universidad de Valencia. España.

Frías-Navarro, D. y Pascual-Soler, M. (2012). Prácticas del análisis factorial exploratorio (AFE) en la investigación sobre conducta del consumidor y marketing. Suma Psicológica, 19, 47-58.

Frías-Navarro, D., Monterde-i-Bort, H., Navarro-González, N., Molina-Palomer, A., Pascual-Soler, M., Perezgonzalez, J., y Longobardi, C. (2018). Statistics anxiety in university students in assessment situations. 4th International Conference on Higher Education Advances (HEAd'18). Universidad Politécnica de Valencia. Valencia, 2018.

Gal, I., Ginsburg, L., y Schau, C. (1997) Monitoring attitudes and beliefs in statistics education. En I. Gal \& J. B. Garfield (Eds.), The assessment challenge in statistics education (pp. 3751). Netherlands IOS.

González, A., Rodríguez, Y., Faílde, J. M., y Carrera, M. V. (2016). Anxiety in the statistics class: Structural relations with self-concept, intrinsic value, and engagement in two samples of undergraduates. Learning and Individual Differences, 45, 214-221.

Gould, R. (2010). Statistics and the modern student. International Statistical Review, 78, 297315.

Kesici, Ş., Baloğlu, M., y Deniz, M. E. (2011). Self-regulated learning strategies in relation with statistics anxiety. Learning and Individual Differences, 21, 472-477. 
Kroenke, K, Spitzer, R. L., y Williams, J. B. (2003). The Patient Health Questionnaire-2: Validity of a two-item depression screener. Medical Care, 41, 1284-1292.

Leadbeater, B., Thompson, K., y Gruppuso, V. (2012). Co-occurring trajectories of symptoms of anxiety, depression, and oppositional defiance from adolescence to young adulthood. Journal of Clinical Child Adolescence Psychology, 41, 719-730.

Lobo, A. y Campos, R. (1997). Los trastornos de ansiedad en atención primaria. Madrid: EMISA.

Macher, D., Paechter, M., Papousek, I., Ruggeri, K., Freudenthaler, H. H., y Arendasy, M. (2013). Statistics anxiety, state anxiety during an examination, and academic achievement. British Journal of Educational Psychology, 83, 535-549.

Macher, D., Paechter, M., Papousek, I., y Ruggeri, K. (2011). Statistics anxiety, trait anxiety, learning behavior, and performance. European Journal of Psychology Education, 27, 483498.

Martínez-Otero, V. (2014). Ansiedad en estudiantes universitarios: estudio de una muestra de alumnos de la facultad de educación. Ensayos, Revista de la Facultad de Educación de Albacete, 29, 63-78.

Misra, R. y Castillo, L. G. (2004). Academic stress among college students: Comparison of American and international students. International Journal of Stress Management, 11, 132-148.

Muñoz, F. J. (2004). El estrés académico. Problemas y soluciones desde una perspectiva psicosocial. Huelva: Servicio de Publicaciones de la Universidad de Huelva.

Murtonen, M. (2005). University students' research orientations: Do negative attitudes exist toward quantitative methods? Scandinavian Journal of Educational Research, 49, 263280.

Murtonen, M. y Lehtinen, E. (2003). Difficulties experienced by education and sociology students in quantitative methods courses. Studies in Higher Education, 28, 171-185.

Nielsen, T. y Kreiner, S. (2018). Measuring statistical anxiety and attitudes toward statistics: The development of a comprehensive Danish instrument (HFS-R). Cogent Education, 5, 1-19.

Onwuegbuzie, A. J. (1997). Writing a research proposal: The role of library anxiety, statistics anxiety, and composition anxiety. Library \& Information Science Research, 19, 5- 33.

Onwuegbuzie, A. J. y Daley, C. E. (1999). Perfectionism and statistics anxiety. Personality and Individual Differences, 26, 1089-1102.

Onwuegbuzie, A. J. y Seaman, M. A. (1995). The effect of time constraints and statistics test anxiety on test performance in a statistics course. Journal of Experimental Education, 62, 115-124.

Onwuegbuzie, A. J. y Wilsom, V. A. (2003). Statistics anxiety: Nature, etiology, antecedents, effects, and treatments: A comprehensive review of the literature. Teaching in Higher Education, 8, 195-209.

Onwuegbuzie, A. J., DaRos, D., y Ryan, J. (1997). The components of statistics anxiety: A phenomenological study. Focus on Learning Problems in Mathematics, 19, 11-35. 
Onwuegbuzie, A., y Wilson, V. (2003). Statistics anxiety: nature, etiology, antecedents, effects, and treatments - a comprehensive review of the literature. Teacher in Higher Education, 8, 195-209.

Papanastasiou, E. C. y Zembylas, M. (2008) Anxiety in undergraduate research methods courses: its nature and implications. International Journal of Research \& Method in Education, 31, 155-167.

Pekrun, R. (2000). A social cognitive, control-value theory of achievement emotions. En J. Heckhausen (Ed.), Motivational psychology of human development. Oxford, UK: Elsevier.

Pekrun, R. (2006). The Control-Value Theory of achievement emotions: Assumptions, corollaries, and implications for educational research and practice. Educational Psychology Review, 18, 315-341.

Pulido, M. A., Serrano, M. L., Valdés, E., Chávez, M. T., Hidalgo, P., y Vera, F. (2011). Estrés académico en estudiantes universitarios. Psicología y Salud, 21, 31-37.

Putwain, D. W., Connors, L., y Symes, W (2010) Do cognitive distortions mediate the test anxietyexamination performance relationship? Educational Psychology, 30, 11-26.

Sandoz, E. K., Butcher, G., y Protti, T. A. (2017). A Preliminary examination of willingness and importance as moderators of the relationship between statistics anxiety and performance. Journal of Contextual Behavioral Science, 6, 47-52.

Spangler, G., Pekrun, R., Kramer, K., y Hofmann, H. (2002). Students' emotions, physiological reactions, and coping in academic exams. Anxiety, Stress \& Coping: An International Journal, 15, 413-432.

Spitzer, R. L., Kroenke, K., Williams, J. B. W., y Löwe, B. (2006). A brief measure for assessing generalized anxiety disorder. Archives of Internal Medicine, 166, 1092-1097.

Stallman, H. M. (2010). Psychological distress in university students: A comparison with general population data. Australian Psychologist, 45, 249-257.

Stallman, H. M., Ohan, J. L., y Chiera, B. (2019). Reducing distress in university students: A randomised control trial of two online interventions. Australian Psychologist, 54, 125-131.

Tosevski, D. L., Milovancevic, M. P., y Gajic, S. D. (2010). Personality and psychopathology of university students. Currents Opinion in Psychiatry, 23, 48-52.

Tran, A., Tran, L., Geghre, N., Darmon, D., Rampal, M., Brandone, D., Gozzo, J. M., Haas, H., Rebouillat-Savy, K., Caci, H., y cols. (2017). Health assessment of French university students and risk factors associated with mental health disorders. PLoS One, 12:e0188187.

Vigil-Colet, A., Lorenzo-Seva, U., y Condon, L. (2008). Development and validation of the Statistical Anxiety Scale. Psicothema, 20, 174-180.

von der Embse, N., Jester, D., Roy, D., y Post, J. (2018). Test anxiety effects, predictors, and correlates: A 30-year meta-analytic review. Journal of Affective Disorders, 227, 483-493.

World Medical Association (2013). World Medical Association Declaration of Helsinki: ethical principles for medical research involving human subjects. JAMA, 310, 2191-2194.

Zeidner, M. (1990). Does anxiety bias scholastic aptitude test performance by gender and sociocultural group? Journal of Genetical Psychology, 150, 175-185. 
Zeidner, M. (2007). Test anxiety in educational contexts. En P. A. Schutz \& R. Pekrun (Eds.), Emotion in education (pp. 165-184). Burlington, MA: Elsevier.

Zeidner, M. (2014). Anxiety in education. En P. A. Alexander, R. Pekrun, \& L. Linnembrik-García (Eds.), International handbook of emotions in education (pp. 265-288). New York, NY: Routledge. 